\title{
Characterisation of Timepix3 with 3D sensor
}

DOI:

10.1088/1748-0221/13/12/C12021

\section{Document Version}

Accepted author manuscript

Link to publication record in Manchester Research Explorer

\section{Citation for published version (APA):}

Gao, T., Da Via, C., Bergmann, B., Burian, P., \& Pospisil, S. (2018). Characterisation of Timepix3 with 3D sensor. Journal of Instrumentation, 13(12), [C12021]. https://doi.org/10.1088/1748-0221/13/12/C12021

\section{Published in:}

Journal of Instrumentation

\section{Citing this paper}

Please note that where the full-text provided on Manchester Research Explorer is the Author Accepted Manuscript or Proof version this may differ from the final Published version. If citing, it is advised that you check and use the publisher's definitive version.

\section{General rights}

Copyright and moral rights for the publications made accessible in the Research Explorer are retained by the authors and/or other copyright owners and it is a condition of accessing publications that users recognise and abide by the legal requirements associated with these rights.

\section{Takedown policy}

If you believe that this document breaches copyright please refer to the University of Manchester's Takedown Procedures [http://man.ac.uk/04Y6Bo] or contact uml.scholarlycommunications@manchester.ac.uk providing relevant details, so we can investigate your claim.

\section{OPEN ACCESS}




\title{
Characterisation of Timepix3 with 3D sensor
}

\author{
T. Gao, ${ }^{a}$ C. Da Via, ${ }^{a}{ }^{1}$ B. Bergmann, ${ }^{b}$ P. Burian ${ }^{b, c}$ and S. Pospisil ${ }^{b}$ \\ ${ }^{a}$ School of Physics and Astronomy, The University of Manchester \\ Oxford Rd, Manchester, Great Britain \\ ${ }^{b}$ Institute of Experimental and Applied Physics, Czech Technical University, \\ Horska 3a/22, Prague, Czech Republic \\ ${ }^{c}$ Faculty of Electrical Engineering, University of West Bohemia, \\ Univerzitni 8, Pilsen, Czech Republic \\ E-mail: Cinzia.DaVia@manchester.ac.uk
}

Abstract: Tests were performed at the SPS facilities at CERN using a $40 \mathrm{GeV} / \mathrm{c}$ pion beam with prototype 3D-Timepix3 detectors (3D detector). A planar-Timepix3 (planar detector) was placed along the beam axis together with the 3D detectors in a telescope arrangement for comparison and reference. We demonstrate that the combination of 3D-geometry silicon sensors and Timepix 3 module can reduce the effect of charge sharing and lowers the carrier drift-time, while giving the same spectroscopy performance without sacrificing the timing or any performance advantages of the Timepix 3 module.

Keywords: Detector control systems (detector and experiment monitoring and slow-control systems, architecture, hardware, algorithms, databases); Performance of High Energy Physics Detectors;

\footnotetext{
${ }^{1}$ Corresponding author.
} 


\section{Contents}

1 Introduction 1

2 Experimental setup 2

3 Method 2

4 Radioactive source and X-ray fluorescence 3

5 Result and analysis of the $40 \mathrm{GeV} / \mathrm{c}$ pion test beam 5

5.1 Cluster size and charge sharing 5

$\begin{array}{lll}5.2 & \text { Particle track samples } & 7\end{array}$

5.3 Energy spectra 8

5.4 Drift-time 9

6 Conclusion 10

\section{Introduction}

The combination of a Timepix 3 readout chip with a solid state sensor as a hybrid active pixel detector $[1,2]$ (Timepix 3 detector), over its past three iterations have been applied in a large number of applications, such as particles tracking, identification, and trajectory mapping [3]. The readout chips were developed within the Medipix Collaboration at CERN. A Timepix3 detector (shown in figure 1) enables simultaneous measurement of Time-over-Threshold (ToT) and Time-of-Arrival (ToA) in every pixel with a time resolution of $1.5625 \mathrm{~ns}$. The chip has a dead time per pixel of approximately $475 \mathrm{~ns}$. It consists of 65,536 pixels in a $2 \mathrm{~cm}^{2}$ area. The module can be operated with a data-driven readout scheme.

Current Timepix detectors are used in conjunction with planar geometry sensors using materials such as silicon, gallium arsenide, cadmium telluride, and other substrates. In conventional planar geometry, the electrodes are at the top and bottom of the detector, with electric fields perpendicular to the plate surface. 3D silicon sensors [4] have arrays of columnar electrodes penetrating through the bulk, with electric fields parallel to the surface (shown in figure 2). 3D geometry decouples the inter-electrode spacing with the sensor thickness which gives this new breed of sensors many advantages over the traditional planar sensor, such as greater radiation tolerance, reduced depletion voltage, and lower carrier collection time. The four electrodes that form a single pixel also act as a "cage" for the induced charge cloud which significantly reduces the charge sharing effect.

With the emergence and usage in particle detection applications of 3D sensors, we decided to investigate the performance of 3D sensors with Timepix 3 modules. Specifically charge sharing, minimum ionising particle charge collection, and charge carrier drift-time. A planar Timepix 3 detector was used as the benchmark for comparison. 


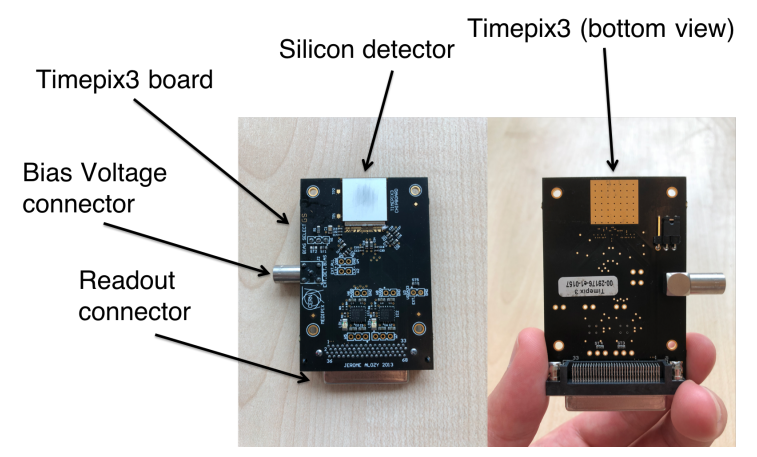

Figure 1: Front and back photos of the Timepix 3 module with 3D sensor mounted. The 3D silicon sensor visible at the top-centre of the two pictures is about $2 \mathrm{~cm}^{2}$. In the picture one can also see the bias voltage lemo-connector and the multi-pin readout connector at the opposite side of the sensor.

\section{Experimental setup}

For this experiment we used Timepix 3 from the Medipix 3 collaboration. The silicon sensor is bump-bonded onto the readout ASIC. This ASIC reads out the induced current from the silicon sensor after a particle penetrates the detector.

The 3D silicon sensor used for this experiment was fabricated at the Institute of Microelectronics of Barcelona IMB-CNM. Its substrate is $300 \mu m$ thick with $227 \mu m$ electrode length, and interelectrode spacing of $55 \mu \mathrm{m}$, as shown in Figure 2. Figure 2a is a microscopic photo showing the cutaway view of the sensor with n-type electrodes partially penetrating the substrate, and figure $2 \mathrm{~b}$ is a sketch of the electrode geometry. The planar sensor used for comparison is $500 \mu m$ thick.

The readout interface, Katherine [5], has a bandwidth of $16 \mathrm{Mhit} / \mathrm{s}$ or $16 \mathrm{Mpix} / \mathrm{s}$ with a Gigabit Ethernet interface. Timepix 3 has the capability to work in self-trigger mode with a set threshold low (THL) value. THL for the 3D detector was set to 1515 (energy equivalent of $4.935 \mathrm{keV}$ ).

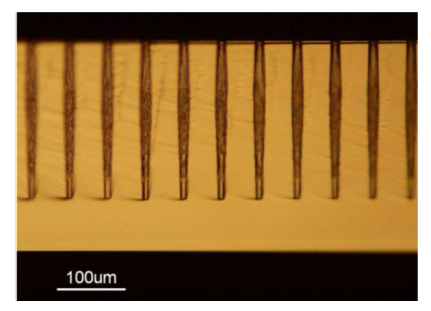

(a)

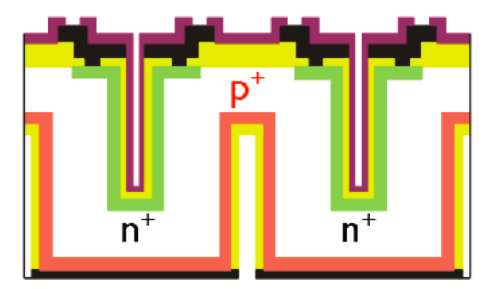

(b) [6]

Figure 2: $2 \mathrm{a}$ is the microscopic photo (taken by fabricators) of the 3D sensor's structure with electrodes. $2 b$ is the illustration of the partial-3D structure.

\section{Method}

3D (named G10 and G11) and planar (named F03) Timepix3 detectors were placed in the pion beam $(40 \mathrm{GeV} / \mathrm{c})$ at the Super-Proton-Synchrotron (SPS) facility at CERN. The detectors were mounted 
on precision rotation motors to change the angle of individual devices relative to the beam. All devices were energy calibrated as described in [7]. For this analysis, the bias voltage for the 3D sensor G10 was set to 20V, and the bias voltage for the planar sensor F03 was 150V.

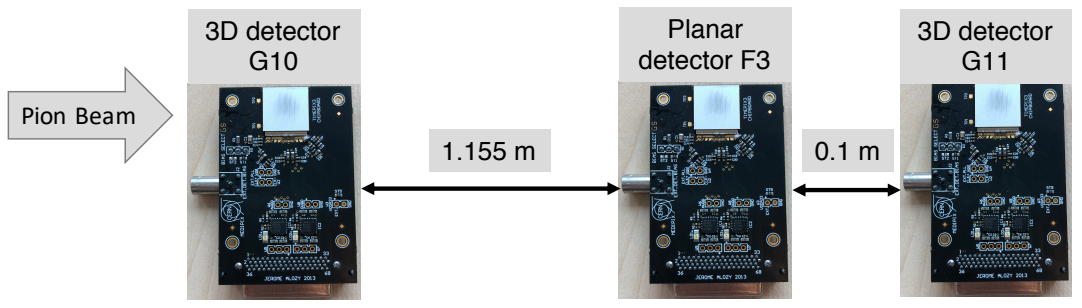

(a)

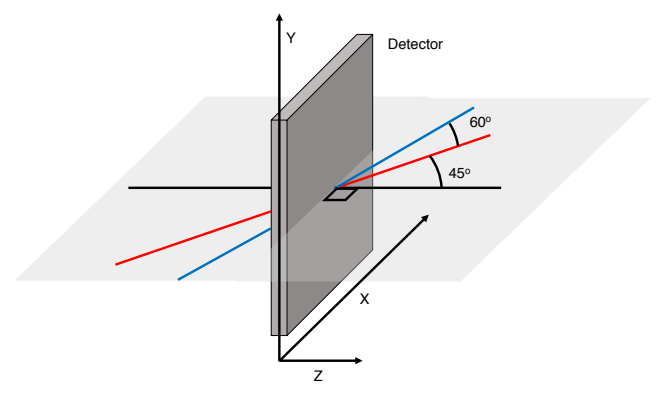

(b)

Figure 3: $3 \mathrm{a}$ is the detector arrangement in the beam line. $3 \mathrm{~b}$ is the orientation of the detector (grey block) with respect to the beam. Where black, red, and blue lines represent the beams with incident angle of $0^{\circ}, 45^{\circ}$, and $60^{\circ}$ respectively. Same coordinate system is used in figure 8 , where $\mathrm{x}$ corresponds to the axis "Pixel No. X" and y to "Pixel No. Y".

The output data from the readout is in text format, which includes the pixel coordinate, ToA, and ToT. The pixel data is clustered as demonstrated in [8] with the time window set to $300 \mathrm{~ns}$ for 3D and $50 \mathrm{~ns}$ for planar ${ }^{1}$. The ToT for this particular track is then summed and scaled according to energy calibration to give the reconstructed particle energy spectroscopy.

\section{Radioactive source and X-ray fluorescence}

The 3D detector was used to measure radioactive sources and X-ray fluorescence from a metal plate for calibration and linearity checks. Radioactive isotopes used were Fe-55 and Am-241, Cadmium plate was used for X-ray fluorescence. Their nominal energy can be found in Table 1. The fitted energy spectrum is shown in figure 4 . In figure 5 a we plotted the measured spectrum peaks against the nominal peaks of each sample to show the accuracy of the energy calibration of the 3D sensor. At higher energy the data points deviate slightly from a straight line fit, however this calibration accuracy is sufficient for the scope of this paper. Figure $4 \mathrm{c}$ and $4 \mathrm{~d}$ indirectly shows that a higher bias voltage has a positive effect on the energy resolution, G11 was fabricated from the same wafer as G10, so they are comparable.

\footnotetext{
${ }^{1}$ The activated pixels with a certain ToA which is within the time window and located next to each other are clustered together and reconstructed to represent a track left behind by a single pion particle.
} 
Table 1: Nominal energy peaks used.

\begin{tabular}{cc}
\hline Source & Energy $(\mathbf{k e V})$ \\
\hline Fe-55 & $5.888 \& 5.899$ \\
Am-241 & 59.5412 \\
Cadmium & $23.173 \& 26.093$ \\
\hline
\end{tabular}

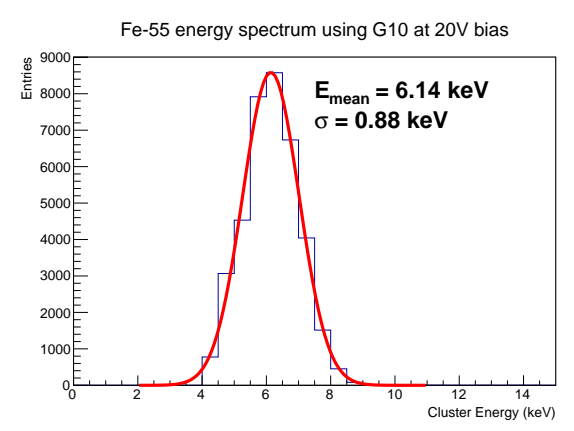

(a) Fe-55 spectrum with G10 at $20 \mathrm{~V}$.

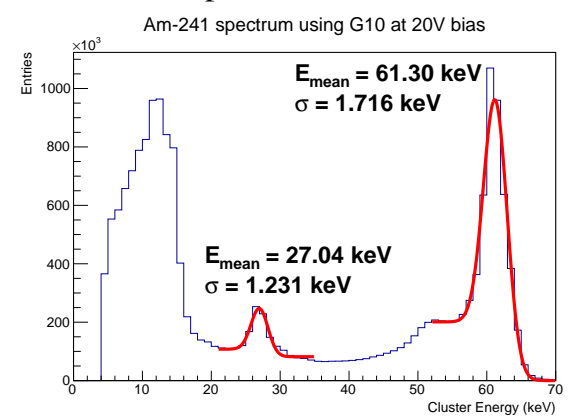

(c) Am-241 spectrum with G10 at $20 \mathrm{~V}$.

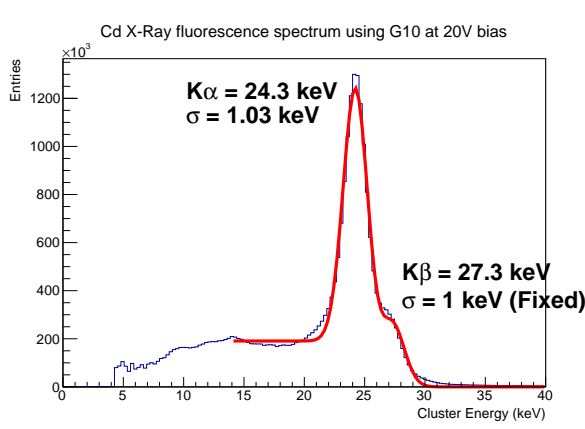

(b) Cd spectrum with G10 at $20 \mathrm{~V}$.

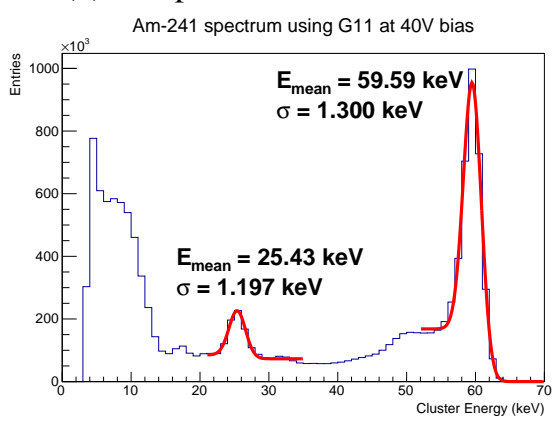

(d) Am-241 spectrum with G11 at $40 \mathrm{~V}$.

Figure 4: Fitted plots of Fe-55 and Am-241 radioactive source and Cadmium X-ray fluorescence. The fit functions used are (4.1), (4.2), and (4.3) respectively, where A, B, and C are constants (number of entries), $\mu$ is the mean of the distribution, $\sigma$ is the standard deviation. The fit results are in the figure. G10 bias voltage were set to $20 \mathrm{~V}$ and G11 bias voltage was set to $40 \mathrm{~V}$.

$$
\begin{aligned}
a & =A \exp \left(-\frac{(x-\mu)^{2}}{2 \sigma^{2}}\right) \\
b & =A \exp \left(-\frac{(x-\mu)^{2}}{2 \sigma^{2}}\right)+\frac{B}{2}\left(1-\operatorname{erf}\left(\frac{x-\mu}{\sqrt{2} \sigma}\right)\right)+C \exp \left(-\frac{\left(x_{2}-\mu_{2}\right)^{2}}{2 \sigma_{2}^{2}}\right) \\
c / d & =A \exp \left(-\frac{(x-\mu)^{2}}{2 \sigma^{2}}\right)+\frac{B}{2}\left(1-\operatorname{erf}\left(\frac{x-\mu}{\sqrt{2} \sigma}\right)\right)
\end{aligned}
$$




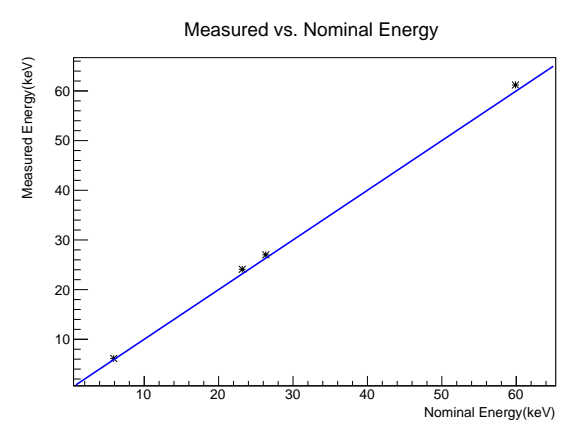

(a)

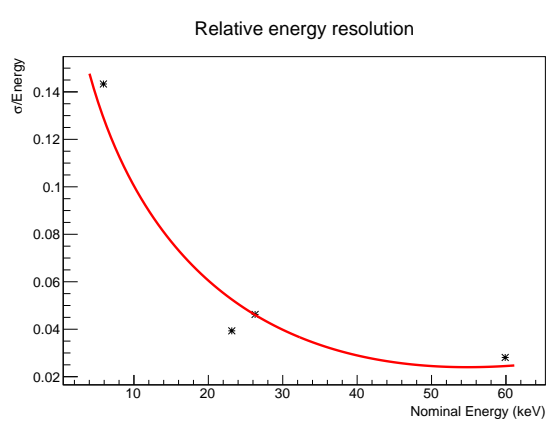

(b)

Figure 5: Measured energy peaks of the radioactive and fluorescence samples against their respective nominal energy peaks. The fit function for figure $5 \mathrm{a}$ is made to fit through zero. The relative energy resolution shows a clear $1 / \sqrt{E}$ relationship.

\section{Result and analysis of the $40 \mathrm{GeV} / \mathrm{c}$ pion test beam}

\subsection{Cluster size and charge sharing}

The experimental setup was described in Sections 2 and 3. The cluster size is the number of pixels grouped together to form the reconstructed track of the particle. A larger beam incident angle creates longer tracks, due to the pion penetrating more pixels. As expected, the 3D detector has a smaller cluster size compared to the planar as shown in figure 6 .

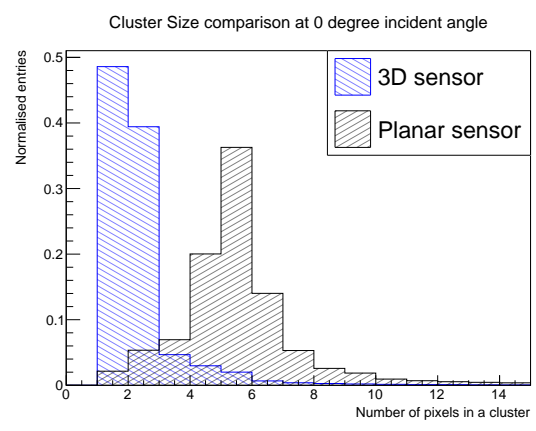

(a) $0^{\circ}$

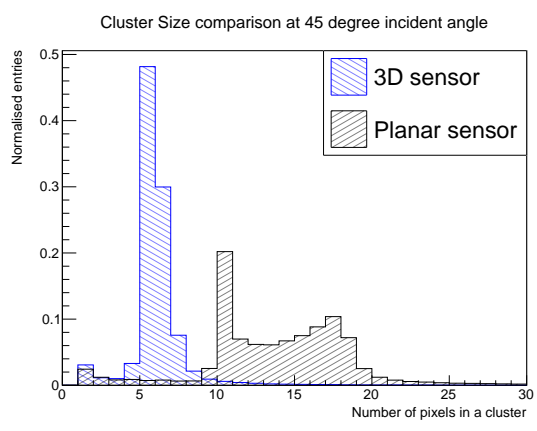

(b) $45^{\circ}$

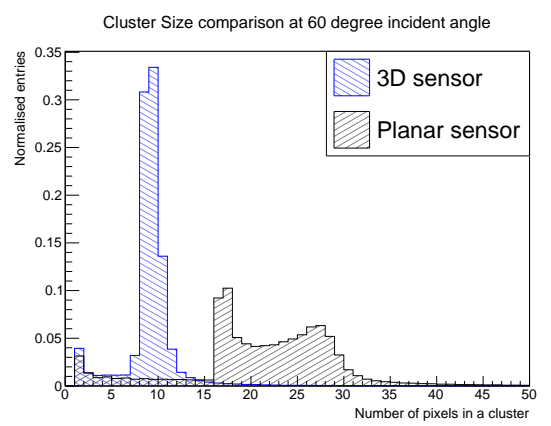

(c) $60^{\circ}$

Figure 6: 3D and planar cluster size comparison. 


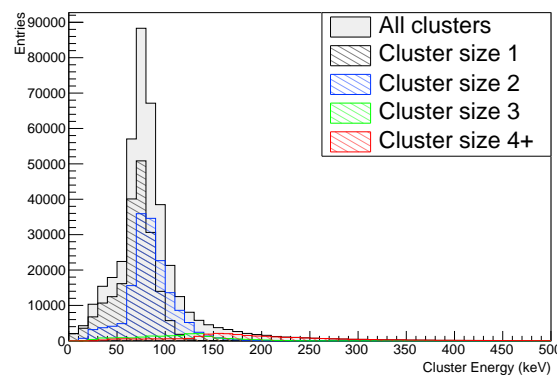

(a) $3 \mathrm{D}$ at $0^{\circ}$

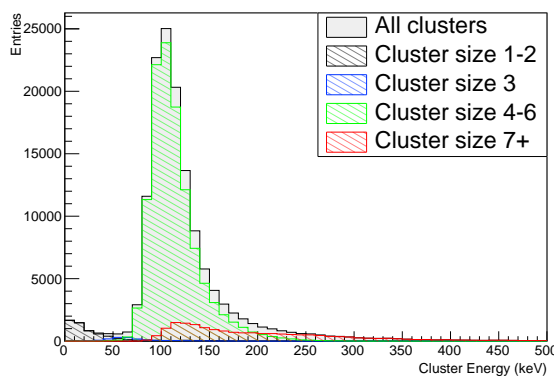

(c) $3 \mathrm{D}$ at $45^{\circ}$

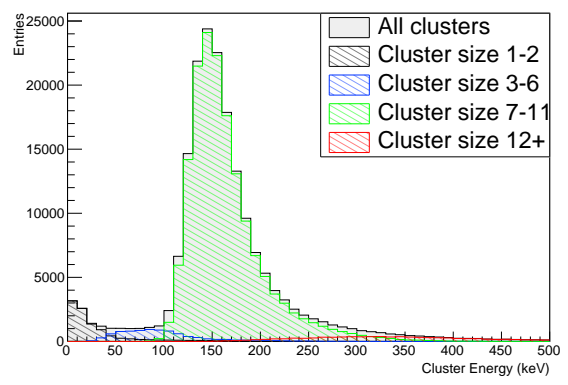

(e) $3 \mathrm{D}$ at $60^{\circ}$

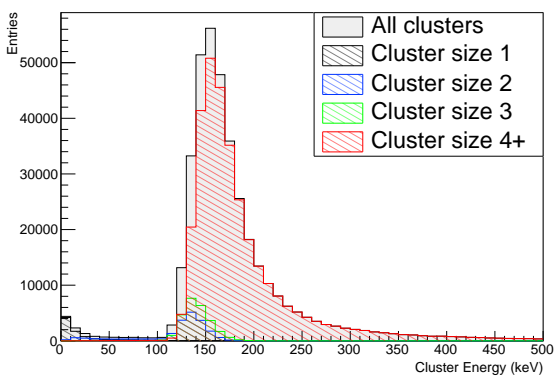

(b) Planar at $0^{\circ}$

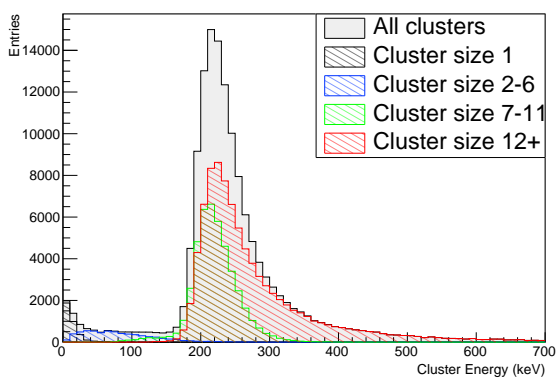

(d) Planar at $45^{\circ}$

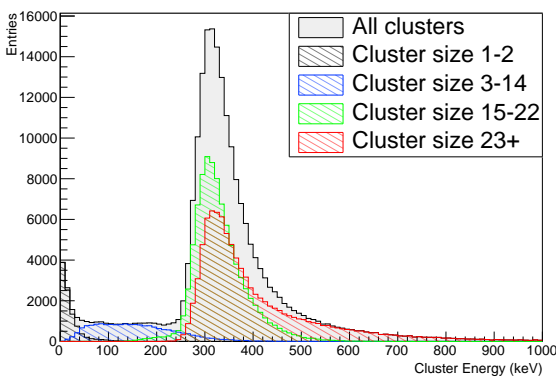

(f) Planar at $60^{\circ}$

Figure 7: 3D and planar detectors at different beam incident angles, with the energy spectrum for each cluster size superimposed.

The reconstructed energy of each different cluster sizes is overlaid on top of the uncut energy spectrum for each incident angle. Figure 7 shows how, at different incident angles, the 3D and planar cluster size combinations contribute to the particle energy spectrum. The energy deposition of minimum ionising particles in matter follows Landau distribution. The substandard background signals below the Landau peak were eliminated by cutting the cluster size variable, exact methods are discussed later. From figure 6 and 7 one can see that it is possible to use the cluster size to cut for every scenario apart from $3 \mathrm{D}$ at $0^{\circ}$ incident angle, where the non-Landau part consisting of 1 pixel clusters and the main pion peak have merged $^{2}$. It should be noted that $3 \mathrm{D}$ tracks have less energy due to thinner silicon bulk $(300 \mu \mathrm{m})$ compared to planar $(500 \mu \mathrm{m})$. Planar results were first

${ }^{2}$ The 0-60 keV energy level can be made up of background radiation, inadequate clustering, scattered pions, and other factors. It should be noted they are not detector noise because as shown in figure $4 \mathrm{a}$, the detector is sensitive to $6 \mathrm{keV}$. 
presented in [9].

\subsection{Particle track samples}

Figure 8 shows the 2D plots of the pixel array, they give a visual representation of how the cluster size changes relative to the beam incident angle. Particles are moving in the negative $\mathrm{x}$ direction.
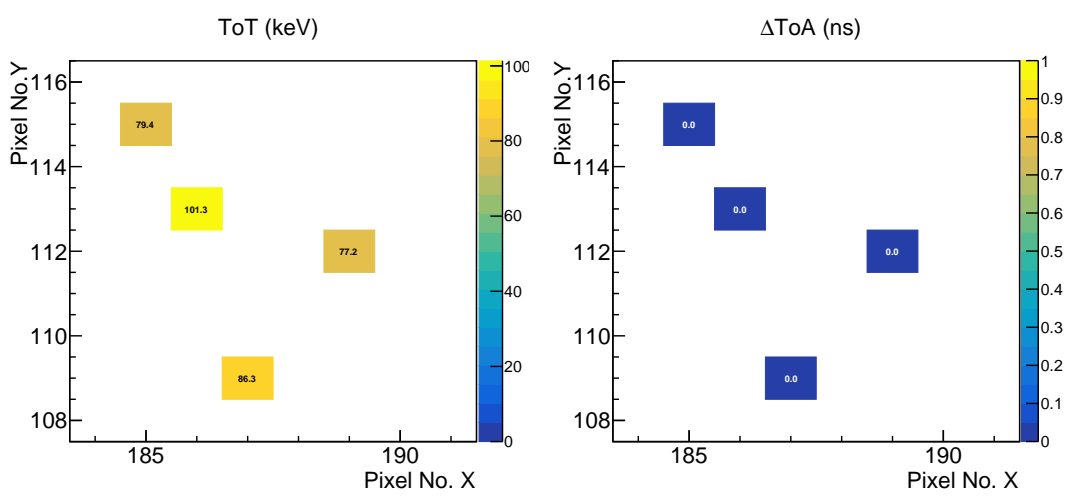

(a) $0^{\circ}$
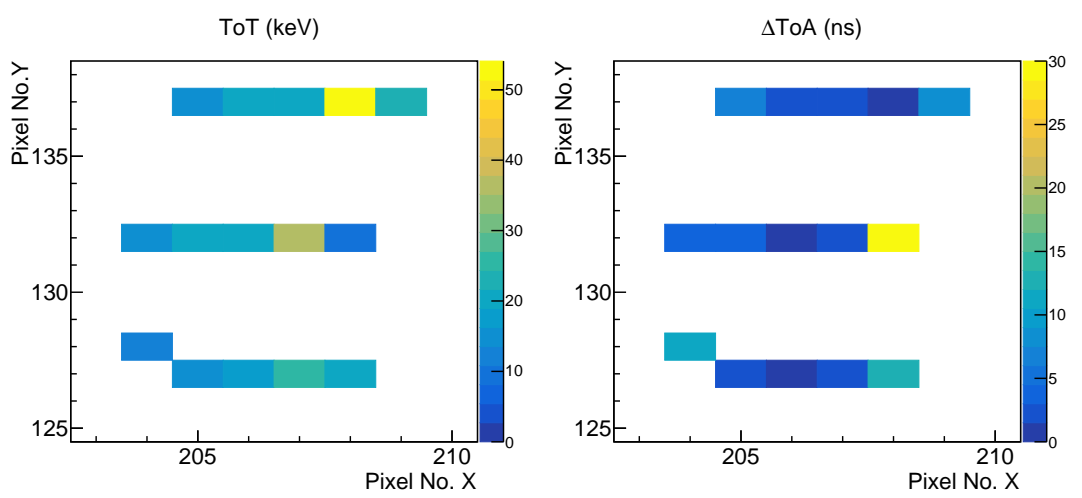

(b) $45^{\circ}$
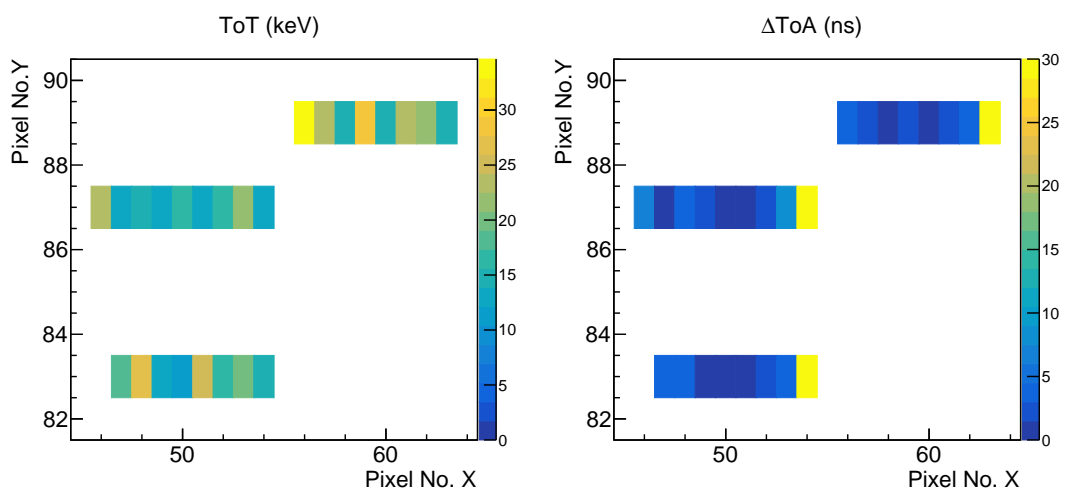

(c) $60^{\circ}$

Figure 8: $3 \mathrm{D}$ detector 2D plots. Left is energy (Colour scale in $\mathrm{keV}$ ) and right is $\Delta \mathrm{ToA}$ (Colour scale in ns). The coordinate system follows figure $3 b$. Pixel No. $\mathrm{X}$ is the pixel number in the $\mathrm{x}$-axis, and same for the y-axis. 
At $0^{\circ}$, the pions only activate a single or double pixel footprint on the $3 \mathrm{D}$ detector. At $45^{\circ}$ and $60^{\circ}$, the pions start to leave a single pixel wide track. Not shown here, planar detectors show 5 pixel clusters in the form of a cross at $0^{\circ}$ and noticeably longer and wider tracks at $45^{\circ}$ and $60^{\circ}$.

In a single cluster, the $\Delta T o A$ is the time difference between the ToA of a pixel and the minimum ToA of the cluster. ToA is the sum of the carrier drift-time and the Timepix 3 time resolution of the particular event. In the $45^{\circ}$ and $60^{\circ}$ plots, the first pixel in some of the tracks has abnormally large $\Delta \mathrm{ToA}$, this phenomenon will be discussed further in Section 5.4.

\subsection{Energy spectra}

The energy deposited by minimum ionising particles (MIPs) in silicon detector is given by the Landau distribution. We determine the most probable value (MPV) and the full width at half maximum (FWHM) of the Landau for both the 3D and the planar detectors at different angles. The 3D and the planar detectors have different thickness, $300 \mu \mathrm{m}$ and $500 \mu \mathrm{m}$ respectively, the FWHM is affected by the thickness of the detector [9].

All cuts were applied to cluster size, except for $3 \mathrm{D}$ detector at $0^{\circ}$ angle where the cut was applied to energy spectrum.

The cuts used for this analysis are given in Table 2, which were derived from previous sections, especially figures 6 and 7 . For $0^{\circ}$, the planar detector gives mainly $\geq 2$ pixels sized clusters, thus the cut is chosen to be cluster size $>1$. The $3 \mathrm{D}$ detector has both 1 and 2 pixel sized clusters in the energy spectrum, hence we cannot use the cluster size cut. Using a GEANT4 simulation we concluded that cutting at energy of $>60 \mathrm{keV}$ matches the expected energy spectrum of the beam.

For $45^{\circ}$, the planar detector with cluster sizes of 1-8 do not contribute to the energy spectrum, as can be seen from figure $7 \mathrm{~d}$ and $6 \mathrm{~b}$, the cut was chosen to be $>8$. For $3 \mathrm{D}$ it is clear from figure $7 \mathrm{c}$ that almost the entire Landau spectrum is made up of cluster size bigger than 4 , hence the cut is chosen to be $>4$.

For $60^{\circ}$, the planar detector has little to no entries in the range of $1-15$ cluster size in figure $6 \mathrm{c}$, the cut is chosen to be $>14$. The $3 \mathrm{D}$ also has negligible number of entries in the range of 1-7 cluster size and figure 7 e further illustrates that all signals lie above cluster size 6 , so the cut is chosen to be $>7$.

Table 2: Final cut selected for each detector geometry and detector incident angle to the beam.

\begin{tabular}{cccc}
\hline Detector Geometry & \multicolumn{3}{c}{ Incident Angle } \\
\cline { 2 - 4 } & $0^{\circ}$ & $45^{\circ}$ & $60^{\circ}$ \\
\hline Planar & $>1$ pixels per cluster & $>8$ pixels per cluster & $>14$ pixels per cluster \\
3D & $>60 \mathrm{keV}$ & $>4$ pixels per cluster & $>7$ pixels per cluster \\
\hline
\end{tabular}




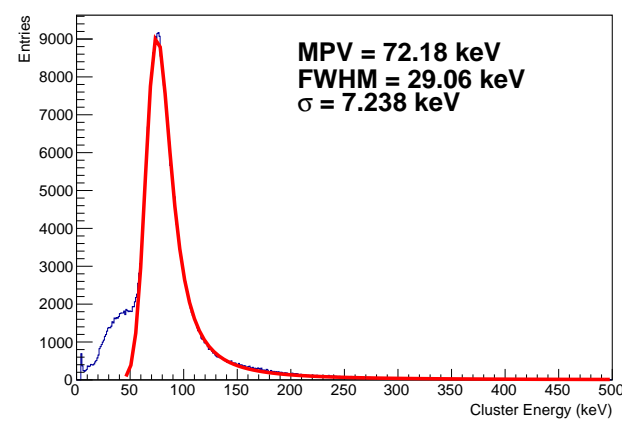

(a) $3 \mathrm{D}$ at $0^{\circ}$

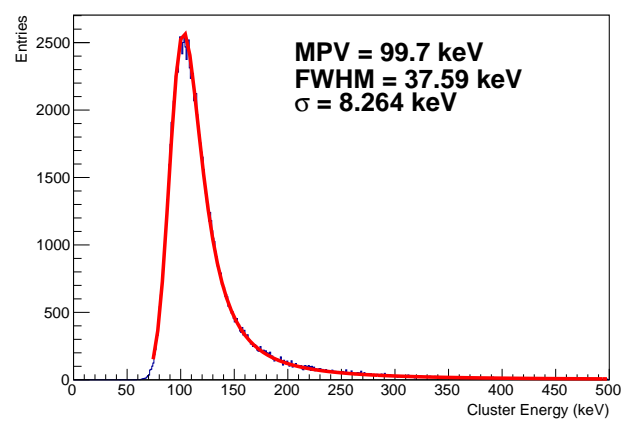

(c) $3 \mathrm{D}$ at $45^{\circ}$

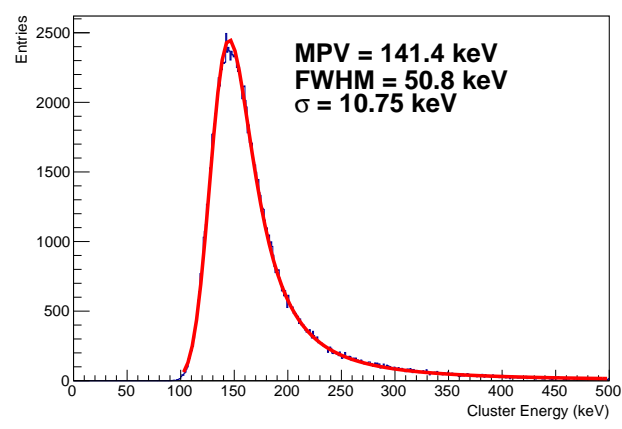

(e) $3 \mathrm{D}$ at $60^{\circ}$

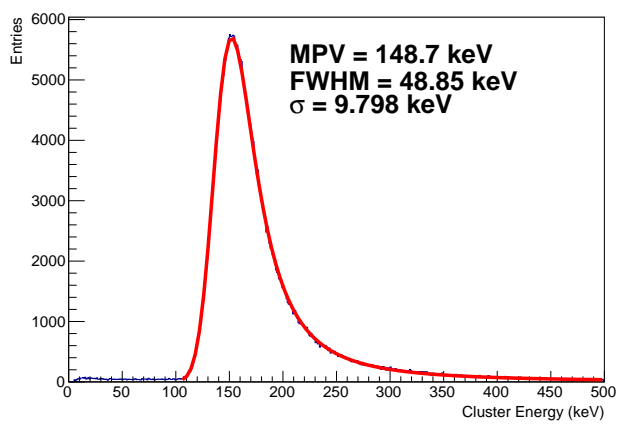

(b) $2 \mathrm{D}$ at $0^{\circ}$

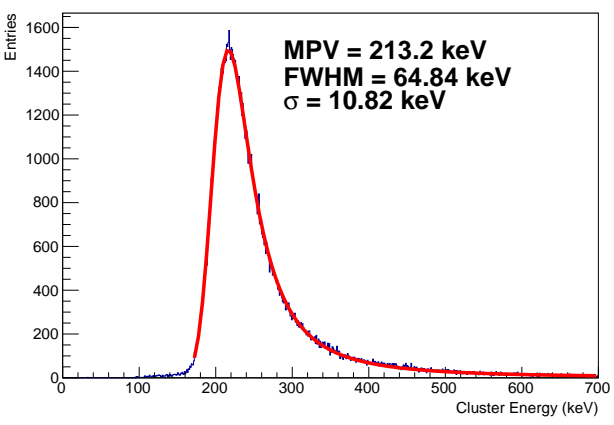

(d) $2 \mathrm{D}$ at $45^{\circ}$

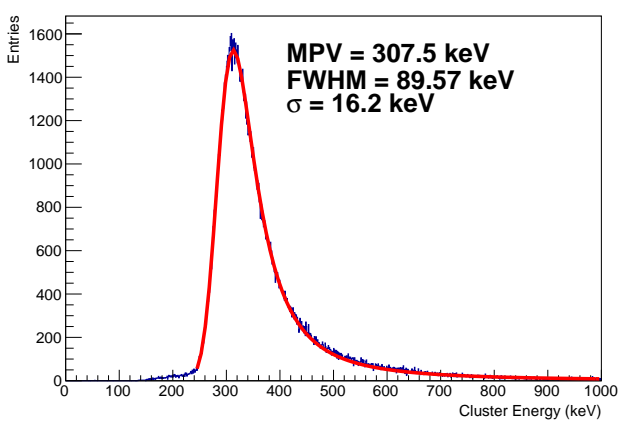

(f) $2 \mathrm{D}$ at $60^{\circ}$

Figure 9: results from different beam incident angles. 3D on the left and planar on the right. Fitted to Convoluted Landau and Gaussian fitting function (Based on a Fortran code by R.Fruehwirth) with FWHM and MPV of the fitted curve displayed.

\subsection{Drift-time}

Drift-time (or signal collection time) is the time taken by the charge carriers to travel to the electrodes from their original creation position. In traditional planar geometry, depending on the carrier creation location, they could travel the entire thickness of the detector. The drift-time for the planar detector can be found in [8], where at $150 \mathrm{~V}$ bias voltage, the maximum drift-time is found to be approximately $30 \mathrm{~ns}$. In 3D geometry, carriers only have to traverse half of the inter-electrode spacing $(27.5 \mu \mathrm{m})$. As shown in figure 10 the $\Delta$ ToA for $68 \%$ of all pixels $(1 \sigma)$ is less than 3.925 


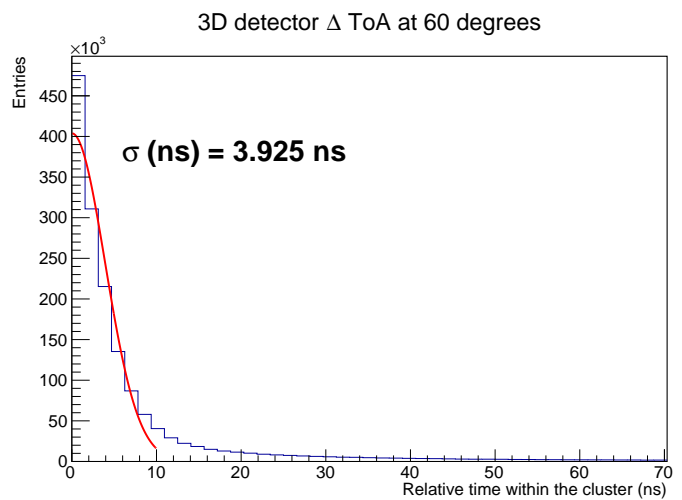

Figure 10: $\Delta \mathrm{ToA}$ spectrum of $3 \mathrm{D}$ detector at $60^{\circ}$ beam incident angle. Fitted with Gaussian distribution with $\mu=0$.

ns. Since the Timepix 3 time resolution is $1.5625 \mathrm{~ns}$, the actual drift time for $68 \%$ of all induced charge carriers is $<3.925 \pm 1.5625 \mathrm{~ns}$.

One abnormality is the high drift-time in the $3 \mathrm{D}$ detector at the start of long clusters. The particles enter the detector from its backside. In the case of $45^{\circ}$ and $60^{\circ}$ incident angles, pions induce the charge carriers near the bottom of the first pixel, which is where the electrode does not extend fully through the substrate, as shown in figure $2 \mathrm{~b}$.

\section{Conclusion}

We have demonstrated the 3D detector have considerably less charge sharing compared to traditional planar silicon sensors when used in conjunction with Timepix 3 modules. The cluster size for the 3D detector due to minimum ionising pions is typically a factor 2 smaller than for the planar detectors. The 3D detector also has a significantly lower drift-time due to the shorter distance needed for induced charge carriers to travel. The result shows the 3D detector has a better at two-track resolution in high pile-up situations, reducing post-processing workload required in cases where the sub-pixel resolution $\left(<(55 / \sqrt{12})^{2} \mu m^{2}\right)$ is not needed. These advantages can be looked upon as favourable in applications such as radiation imaging and high energy particle detection.

\section{Acknowledgments}

\section{References}

[1] Llopart, X., Ballabriga, R., Campbell, M., Tlustos, L. and Wong, W., 2007. Timepix, a 65k programmable pixel readout chip for arrival time, energy and/or photon counting measurements. Nuclear Instruments and Methods in Physics Research Section A: Accelerators, Spectrometers, Detectors and Associated Equipment, 581(1-2), pp.485-494.

[2] Poikela, T., Plosila, J., Westerlund, T., Campbell, M., De Gaspari, M., Llopart, X., Gromov, V., Kluit, R., van Beuzekom, M., Zappon, F. and Zivkovic, V., 2014. Timepix3: a $65 \mathrm{~K}$ channel hybrid pixel readout chip with simultaneous ToA/ToT and sparse readout. Journal of instrumentation, 9(05), p.C05013. 
[3] Ballabriga, R., Campbell, M. and Llopart, X., 2018. Asic developments for radiation imaging applications: The medipix and timepix family. Nuclear Instruments and Methods in Physics Research Section A: Accelerators, Spectrometers, Detectors and Associated Equipment, 878, pp.10-23.

[4] Da Via, C., Boscardin, M., Dalla Betta, G.F., Darbo, G., Fleta, C., Gemme, C., Grenier, P., Grinstein, S., Hansen, T.E., Hasi, J. and Kenney, C., 2012. 3D silicon sensors: Design, large area production and quality assurance for the ATLAS IBL pixel detector upgrade. Nuclear Instruments and Methods in Physics Research Section A: Accelerators, Spectrometers, Detectors and Associated Equipment, 694, pp.321-330.

[5] Burian, P., Broulím, P., Jára, M., Georgiev, V. and Bergmann, B., 2017. Katherine: ethernet embedded readout interface for Timepix3. Journal of Instrumentation, 12(11), p.C11001.

[6] Capeans, M., Flick, T., Vuillermet, R., Darbo, G., Pernegger, H., Einsweiller, K., Garcia-Sciveres, M., Elsing, M., Rohne, O. and Gemme, C., 2010. ATLAS insertable B-layer technical design report (No. CERN-LHCC-2010-013). ATLAS-TDR-019.

[7] Jakubek, J., 2011. Precise energy calibration of pixel detector working in time-over-threshold mode. Nuclear Instruments and Methods in Physics Research Section A: Accelerators, Spectrometers, Detectors and Associated Equipment, 633, pp.S262-S266.

[8] Bergmann, B., Pichotka, M., Pospisil, S., Vycpalek, J., Burian, P., Broulim, P. and Jakubek, J., 2017. $3 D$ track reconstruction capability of a silicon hybrid active pixel detector. The European Physical Journal C, 77(6), p.421.

[9] Burian, P., Broulím, P., Georgiev, V., Bergmann, B. and Pospílil, S., 2018. Particle telescope with Timepix3 pixel detectors. Journal of Instrumentation, 13(01), p.C01002.

[10] Groom, D.E. and Klein, S.R., 2000. Passage of particles through matter. The European Physical Journal C-Particles and Fields, 15(1-4), pp.163-173. 